\title{
Indocyanine green as effective antibody conjugate for intracellular molecular targeted photodynamic therapy
}

Sijia Wang

Gereon Hüttmann

Florian Rudnitzki

Heyke Diddens-Tschoeke

Zhenxi Zhang

Ramtin Rahmanzadeh 


\title{
Indocyanine green as effective antibody conjugate for intracellular molecular targeted photodynamic therapy
}

\author{
Sijia Wang, ${ }^{a, b}$ Gereon Hüttmann, ${ }^{b}$ Florian Rudnitzki, ${ }^{b}$ Heyke Diddens-Tschoeke, ${ }^{b}$ Zhenxi Zhang, ${ }^{a}$ and \\ Ramtin Rahmanzadeh ${ }^{\text {b,* }}$ \\ a'Xi'an Jiaotong University, School of Life Science and Technology, Institute of Biomedical Analytical Technology and Instrumentation, \\ Key Laboratory of Biomedical Information Engineering of Ministry of Education, Xi'an 710049, China \\ bUniversity of Lübeck, Institute of Biomedical Optics, Peter-Monnik-Weg 4, Lübeck 23562, Germany
}

\begin{abstract}
The fluorescent dye indocyanine green (ICG) is clinically approved and has been applied for ophthalmic and intraoperative angiography, measurement of cardiac output and liver function, or as contrast agent in cancer surgery. Though ICG is known for its photochemical effects, it has played a minor role so far in photodynamic therapy or techniques for targeted protein-inactivation. Here, we investigated ICG as an antibody-conjugate for the selective inactivation of the protein $\mathrm{Ki}-67$ in the nucleus of cells. Conjugates of the Ki-67 antibody TuBB-9 with different amounts of ICG were synthesized and delivered into HeLa and OVCAR-5 cells through conjugation to the nuclear localization sequence. Endosomal escape of the macromolecular antibodies into the cytoplasm was optically triggered by photochemical internalization with the photosensitizer BPD. The second light irradiation at $690 \mathrm{~nm}$ inactivated Ki-67 and subsequently caused cell death. Here, we show that ICG as an antibody-conjugate can be an effective photosensitizing agent. Best effects were achieved with 1.8 ICG molecules per antibody. Conjugated to antibodies, the ICG absorption peaks vary proportionally with concentration. The absorption of ICG above $650 \mathrm{~nm}$ within the optical window of tissue opens the possibility of selective Ki-67 inactivation deep inside of tissues. ๑ The Authors. Published by SPIE under a Creative Commons Attribution 3.0 Unported License. Distribution or reproduction of this work in whole or in part requires full attribution of the original publication, including its DOI. [DOI: 10.1117/1. JBO.21.7.078001]
\end{abstract}

Keywords: Indocyanine green; Ki-67 protein; antibody conjugate; intracellular delivery; light inactivation; photodynamic therapy; biomedical optics; medicine; targets; cells; infrared.

Paper 160081R received Feb. 9, 2016; accepted for publication Jun. 29, 2016; published online Jul. 18, 2016.

\section{Introduction}

Indocyanine green (ICG) has been used in clinical diagnostics for over 40 years with an excellent safety record. ${ }^{1}$ It has a very broad absorption band between 600 and $800 \mathrm{~nm}$ and a fluorescence emission above $800 \mathrm{~nm}$. In this wavelength range, light absorption and scattering of biological tissue is relatively low and light can penetrate deep into the tissue. Also, autofluorescence of tissue is low at this wavelength range. ${ }^{2,3}$ ICG is approved by the U.S. Food and Drug Administration (FDA) and the European Medicines Agency for ophthalmic and intraoperative angiography, measurement of cardiac output and liver function, and more recently as a contrast agent in cancer related surgery. ${ }^{4}$ Especially, the literature about the use of ICG in cancer diagnosis has grown exponentially in the last years. ${ }^{5}$ Here, ICG is used for Sentinel lymph node mapping in breast cancer, skin cancers, cervical cancer, and enteric cancers, or the detection of metastatic lesions in liver cancer and staging of lung cancers. ${ }^{5,6}$ These in vivo applications are based on the near infrared (NIR) fluorescence of ICG. NIR fluorescence imaging is faster, less expensive, and shows a reduced tendency to miss local metastasis in comparison to computer tomography or positron emission tomography. ${ }^{7}$ ICG has not only been investigated for diagnosis but also as a photosensitizer for photodynamic therapy (PDT).

*Address all correspondence to: Ramtin Rahmanzadeh, E-mail: rahmanzadeh@bmo.uni-luebeck.de
PDT is an approved treatment modality for various malignant and nonmalignant diseases. ${ }^{8,9}$ ICG generates singlet oxygen when exposed to light and damages the surrounding tissue. ${ }^{10}$ Bäumler et al. ${ }^{11}$ have shown that ICG leads to lipid peroxidation upon light irradiation and has a very low-dark toxicity compared with porphyrines. Phototoxic effects of ICG have been studied in different cancer cell lines, originating from breast cancer, ${ }^{12,13}$ pancreatic cancer, ${ }^{14}$ oral squamous cell cancer, ${ }^{15}$ and skin melanoma. ${ }^{16}$ ICG has also been encapsulated in theranostic nanoparticles for tumor imaging or targeted PDT approaches. ${ }^{17,18}$ Liu et al. ${ }^{19}$ encapsulated ICG into micelles with folic acid (FA) targeting specificity where ICG was loaded in such a high concentration that self-quenching occurred. After uptake into FA positive cells, the particles became highly fluorescent and phototoxic after cellular degenerative processes. Kuo et al. ${ }^{20}$ show a combined approach of photothermal therapy and PDT for elimination of malignant cells where ICG was conjugated to gold nanoparticles. Further photochemical active nanoparticles include $\mathrm{TiO}_{2}$ nanoparticles that are under irradiation of UV and visible light capable of photocatalytic degeneration of large molecules as well as Escherichia coli due to decomposition of the lipopolysaccharide in the outer leaflet of the bacteria. ${ }^{21}$

In this study, we use ICG for the inactivation of the nuclear protein $\mathrm{Ki}-67$ with the aim of eliminating proliferating cancer cells. The expression of $\mathrm{Ki}-67$ is strongly correlated with cell proliferation $^{22,23}$ and therefore Ki-67 is an established marker in tumor diagnosis for cell proliferation in histopathology. ${ }^{24}$ In our study, ICG was conjugated to the antibody TuBB-9, 
which specifically recognizes a physiologically active form of $\mathrm{Ki}-67$ that is involved in the early steps of the synthesis of rRNA. $^{25,26}$

In earlier studies, we have shown that light inactivation of Ki67 leads to a selective elimination of proliferating cells. ${ }^{27-29}$ In these studies, we have used fluorescein isothiocyanate (FITC) as a photosensitizer. To study the effectiveness of ICG to inactivate Ki-67, we synthesized conjugates with different ratios of ICG per TuBB-9 antibody. ICG is an amphiphilic molecule and the absorption properties are strongly dependent on the solvent and the ICG concentration. Especially, the first absorption peak at $700 \mathrm{~nm}$ is influenced by concentration effects. ${ }^{30}$ This can lower the efficiency of ICG as a photosensitizer and is challenging for the application of the right light dose for therapeutic treatments. Here, we show that ICG-antibody conjugates can be effective photosensitizing agents. In contrast to free ICG, the absorption peaks vary proportionally with concentration, and wavelength shift is diminished.

For nuclear delivery of the TuBB-9-ICG photoimmunoconjugates, we used a recently developed delivery platform. ${ }^{28}$ First, TuBB-9-ICG was covalently linked to the nuclear localization signal (NLS) peptide sequence for intracellular delivery. In addition, for active nuclear transport, NLS is known to act also as a cell penetrating peptide and proteins labeled with NLS can be transported into cells. ${ }^{31}$ This cellular transport, especially for macromolecules like antibodies, ends often in the endosomal pathway, where the delivered agents are finally degraded in lysosomes, before they can exert their action. ${ }^{32}$

In order to provide endosomal escape of the macromolecular TuBB-9 antibodies into the cytoplasm, we utilized photochemical internalization (PCI) with optical irradiation after incubation of the cells with an appropriate photosensitizer. Lipophilic and amphiphilic porphyrins or porphyrin-related compounds localize primarily in endosome membranes. ${ }^{33}$ Light irradiation can disrupt the membranes and can set their contents free. ${ }^{34} \mathrm{PCI}$ is based on the same working mechanism as PDT. Both techniques utilize photosensitizers for the generation of singlet oxygen and other reactive oxygen species (ROS) upon light irradiation. ${ }^{35}$ In order to restrict the photochemical effect to endosomes, PCI requires lower photosensitizer concentrations and light than PDT. ${ }^{34}$ The short lifetime $(<0.04 \mu \mathrm{s})$ and short range of action $(<20 \mathrm{~nm})^{36}$ of these ROS limits most of the damaging effects to the endosomal membranes. Endosomes can be disrupted with the help of ROS by oxidizing its membrane constituents, which facilitates the escape of trapped therapeutic macromolecules into the cytoplasm. ${ }^{37}$

Here, we incubated HeLa and OVCAR-5 cells with the lipophilic photosensitizer benzoporphyrine derivative MA (BPD) for PCI to deliver the TuBB-9-ICG-NLS constructs to the cell nucleus. The second light irradiation at $690 \mathrm{~nm}$ (for ICG) or $490 \mathrm{~nm}$ (for FITC) leads to the inactivation of $\mathrm{Ki}-67$ or its binding partners.

\section{Materials and Methods}

\subsection{Antibody and Indocyanine Green Labeling}

The monoclonal mouse antibody TuBB-9 was produced from hybridoma cells kindly provided by Leibnitz Institute Borstel, Germany. ${ }^{25}$ Hybridoma cells were cultivated in bioreactor cell culture flasks (INTEGRA Biosciences, Switzerland). The antibodies were purified from the culture supernatant with protein $G$ columns. For labeling with ICG, antibodies $(1 \mathrm{mg} / \mathrm{ml}$ in PBS at
$\mathrm{pH}$ 7.6) were mixed with the ICG-NHS (1.21 mM in PBS at $\mathrm{pH}$ 7.6, Intrace medical, Switzerland) in a molar ratio of $1: 2,1: 4$, and 1:8, respectively. The solution was incubated at room temperature on a shaker for $1 \mathrm{~h}$, and the labeled antibody was purified with a NAP-5 Sephadex column (GE Healthcare). After elution with PBS ( $\mathrm{pH} 7.4$ ), the labeled antibodies were concentrated with Microcon tubes (Millipore) and resuspended in PBS ( $\mathrm{pH}$ 7.4). The absorption spectrum of free ICG, ICGNHS and ICG labeled TuBB-9 (TuBB-9-ICG) was measured by UV-VIS spectroscopy (Hitachi, Japan).

\subsection{Conjugation of TuBB-9-ICG with Nuclear Localization Signal Peptides}

Cysteine modified NLS (CGGGPKKKVED, Anaspec) was conjugated to TuBB-9-ICG using the linker molecule sulfosuccinimidyl-4-(N-maleimidomethyl)-cyclohexane-1-carboxylate (sulfo-SMCC; Pierce). ${ }^{38}$ The maleimide groups were introduced to the conjugates by the reaction of $3 \mathrm{mg}$ TuBB-9-ICG with $0.14 \mathrm{mg}$ sulfo-SMCC in phosphate buffered saline (PBS, pH 7.4) in a ratio of $1: 15$ at room temperature for $1 \mathrm{~h}$. The maleimide-derivatized TuBB-9-ICG was then purified on a NAP5 mini-column (exclusion limit $5 \mathrm{kDa}$; GE Healthcare) eluated with PBS, pH 7.0. The fractions containing maleimide TuBB-9ICG were transferred to an ultrafiltration device (100 kDa cutoff; Amicon). The device was centrifuged at $800 \mathrm{~g}$ for $20 \mathrm{~min}$ to concentrate maleimide-TuBB-9-ICG to $2 \mathrm{mg} / \mathrm{ml}$, which were then mixed with a 60-fold molar excess of NLS-peptides $\left(10 \mathrm{mg} / \mathrm{ml}\right.$ in PBS, pH 7.0) for $18 \mathrm{~h}$ at $4^{\circ} \mathrm{C}$. NLS-TuBB-9ICG conjugates were purified from excess NLS-peptides and concentrated to $2 \mathrm{mg} / \mathrm{ml}$ in PBS ( $\mathrm{pH} 7.4$ ) by ultrafiltration.

NLS-TuBB-9-ICG conjugates were analyzed by sodium dodecyl sulfate-2-polyacrylamide gel electrophoresis (SDSPAGE) under nonreducing conditions on a $5 \%$ Tris- $\mathrm{HCl}$ readyminigel (Bio-Rad) stained with Coomassie Brilliant Blue. The migration distance in the gel relative to the bromphenol blue dye front $\left(R_{\mathrm{f}}\right)$ was measured and the numbers of NLS-peptides bound to the TuBB-9-ICG were estimated. For characterization and concentration measurements the absorption spectra of NLSTuBB-9-ICG conjugates were measured by UV-VIS spectroscopy (Hitachi, Japan) and the emission spectra were recorded by fluorescence spectroscopy (FluoroMax, SPEX).

\subsection{Cell Culture}

Human cervical cancer cell line HeLa and human ovarian adenocarcinoma cell line OVCAR-5 were obtained from American Type Culture Collection. HeLa cells were maintained in Dulbecco's modified Eagle's medium (DMEM low glucose, Sigma) containing 10\% fetal bovine serum (FBS gold, PAA) and $1 \%$ Penicillin/Streptomycin (PAA). OVCAR-5 cells were cultured in RPMI-1640 medium (Sigma) containing 10\% FBS and $1 \%$ Penicillin/Streptomycin. Fibroblast cells were maintained in DMEM medium (High glucose, Sigma) containing $10 \%$ FBS and $1 \%$ Penicillin/Streptomycin. Cells were incubated in humidified atmosphere at $37^{\circ} \mathrm{C}$ with $5 \% \mathrm{CO}_{2}$.

\subsection{Endosomal Release of TUBB-9-ICG-NLS}

HeLa and OVCAR-5 cells were plated in glass bottom imaging dishes $\left(4 \times 10^{4}\right.$ cells $\left./ \mathrm{ml}\right)$ in a medium containing $100 \mathrm{nM} \mathrm{BPD}$ (benzoporphyrin derivative monoacid Ring A, Sigma). After 18 to $20 \mathrm{~h}$, cells were washed twice with PBS and incubated for $4 \mathrm{~h}$ with $(15 \mu \mathrm{g} / \mathrm{ml})$ NLS-TuBB-9-ICG conjugates. Then, the cells 
were washed once with serum-free DMEM and twice with PBS. Light irradiation for endosomal escape was performed with a $410 \mathrm{~nm}$ LED (Roithner Lasertechnik, Vienna, Austria) with $15 \mathrm{~mW} / \mathrm{cm}^{2}$ for $60 \mathrm{~s}$. Untreated cells, treated cells without irradiation, and treated cells with irradiation were fixed with $4 \%$ Paraformaldehyde and permeabilized by $0.25 \%$ Triton, then the fixed cells were incubated with $5 \mu \mathrm{g} / \mathrm{ml}$ Alexa 546 labeled Goat Anti-Mouse IgG Antibody (Life Technologies) for $45 \mathrm{~min}$. Cells were washed twice with PBS and imaged with a fluorescence microscope (Nikon, Japan).

\subsection{Cell Viability Assay}

For viability assay, HeLa and OVCAR-5 cells were seeded with a density of $4 \times 10^{4}$ cells $/ \mathrm{ml}$ in 96-well plates, $100 \mathrm{nM}$ BPD was added into each well, and incubated for $18 \mathrm{~h}$. Then, cells were incubated for another $4 \mathrm{~h}$ with $(15 \mu \mathrm{g} / \mathrm{ml}) \mathrm{NLS}$ TuBB-9-ICG constructs with different ICG ratios (antibodyICG ratios: $1: 0.6,1: 1.8$, and $1: 5.2$ ) and for comparison with NLS-TuBB-9-FITC conjugates. After incubation, the cells were washed twice with PBS and wells were divided into four groups: (1) no light irradiation, (2) irradiation with $410 \mathrm{~nm}$ laser for endosomal escape $\left(15 \mathrm{~mW} / \mathrm{cm}^{2}, 30 \mathrm{~s}\right)$, (3) incubation for $48 \mathrm{~h}$ and irradiation at $690 \mathrm{~nm}$ with a LED for photo-inactivation of $\mathrm{Ki}-67\left(71 \mathrm{~mW} / \mathrm{cm}^{2}, 5 \mathrm{~min}\right)$, and (4) irradiation at $410 \mathrm{~nm}$ and after $48 \mathrm{~h}$ a second irradiation at $690 \mathrm{~nm}$ for endosomal escape and photo-inactivation of ICG. After $72 \mathrm{~h}$, cells were washed twice with PBS and $100 \mu$ l MTT-solution $(1 \mathrm{mg} / \mathrm{ml}$ in culture medium) was added per well. After an additional incubation for $1 \mathrm{~h}$, culture medium was removed and the samples were incubated in $200 \mu 1$ DMSO for $30 \mathrm{~min}$ on a shaker to dissolve the formazan crystals. Absorbance was measured on a microplate reader (Spectramax M5, Molecular Devices) at $570 \mathrm{~nm}$. Absorbance changes of treated samples compared to untreated controls were used as a measure for cell viability. Statistical analysis was performed by using SPSS (IBM Deutschland $\mathrm{GmbH}$ ). The viability data of different groups were expressed as mean value and standard deviation of a total of eight measurements. Student's $t$-test was used and differences were considered significant at $p<0.05$. To establish the growth curves of the different treatment groups three cell samples were counted each day.

\section{Results}

\subsection{Absorption Characteristics of Indocyanine Green and TuBB-9-ICG}

For our study, we prepared TuBB-9-ICG conjugates with three different ratios of antibody to ICG as shown in Table 1 . The

Table 1 TuBB-9-ICG ratios used for conjugation reaction and the ratios after reaction, determined after absorption measurements.

\begin{tabular}{lcc}
$\begin{array}{l}\text { Name of } \\
\text { conjugates }\end{array}$ & $\begin{array}{c}\text { Molar ratio of TuBB-9 } \\
\text { and ICG-NHS used } \\
\text { for conjugation }\end{array}$ & $\begin{array}{c}\text { Measured molar ratio of } \\
\text { TuBB-9 and ICG-NHS } \\
\text { after conjugation }\end{array}$ \\
\hline TuBB-9-ICG 1 & $1: 2$ & $1: 1.8$ \\
TuBB-9-ICG 2 & $1: 4$ & $1: 3.1$ \\
TuBB-9-ICG 3 & $1: 8$ & $1: 6.3$ \\
\hline
\end{tabular}

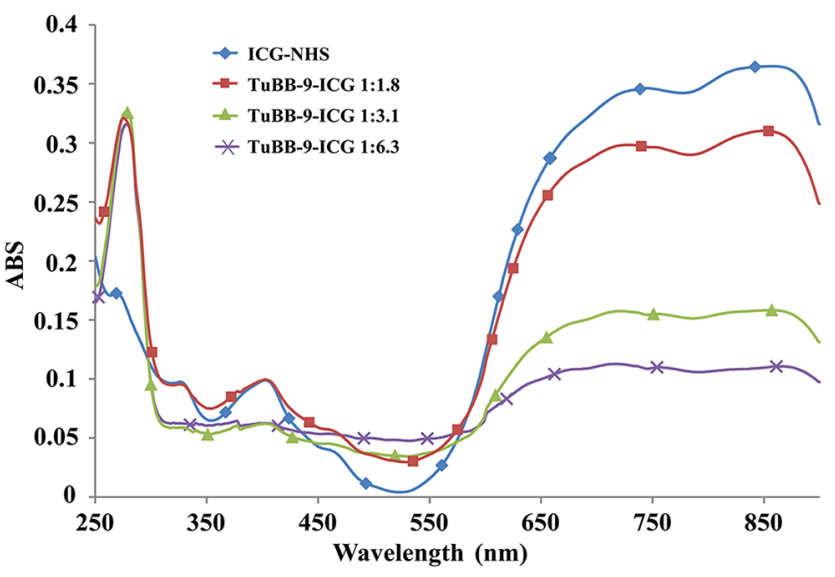

Fig. 1 Absorption spectra of ICG conjugated in different ratios to the antibody TuBB-9. For measurements, samples were adjusted to the same TuBB-9 concentrations and diluted in PBS. The peak at $280 \mathrm{~nm}$ originates from protein absorption of the TuBB-9 antibody.

absorption spectrum of ICG-NHS (used for labeling) and different TuBB-9-ICG conjugates are shown in Fig. 1. ICG-NHS and TuBB-9-ICG conjugates show high absorption in the range from 650 to $900 \mathrm{~nm}$, with two peaks around 730 and $800 \mathrm{~nm}$, which fits the optical window of biological tissue. The peak at $280 \mathrm{~nm}$ originates from protein absorption of the TuBB-9 antibody.

One of the disadvantages for clinical application of ICG is the nonlinear dependence of light absorption on the concentration, because of aggregation. The ratio of the absorption peaks of free ICG at 700 and $770 \mathrm{~nm}$ changes with increasing concentration [Fig. 2(a)]. However, after conjugation to the antibody, the spectral shape remains stable and the two peaks at 730 and $800 \mathrm{~nm}$ tend to increase linearly with concentration [Fig. 2(b)].

\subsection{Conjugation of TuBB-9-ICG with Nuclear Localization Signal Peptides}

In order to increase cellular uptake for intracellular delivery, the three different TuBB-9-ICG conjugates listed in Table 1 were covalently conjugated with the cell penetrating peptide NLS. Figure 3(a) depicts schematically the chemical reaction of the conjugation. The resulting constructs were analyzed by SDSPAGE to estimate the number of NLS-peptides conjugated to each molecule of TuBB-9-ICG conjugate. As shown in Fig. 3(b) after coomassie blue protein staining, the band of NLS-TuBB-9-ICG has a distinctly longer migration distance than TuBB-9-ICG. This difference in moving distance corresponds to different amounts of NLS-peptides conjugated to TuBB-9-ICG. Absorption spectra of the NLS-TuBB-9-ICG constructs showed no change on the absorption peak position of NLS-TuBB-9-ICG and TuBB-9-ICG. Nevertheless, the molar ratio of TuBB-9 and ICG decreased slightly after the NLS conjugation, since ICG and NLS are both connected via NHS groups to the amino groups of TuBB-9 [Fig. 3(c)]. The characterization results are listed in Table 2. Figure 3(d) shows the fluorescence emission spectrum of ICG-NHS, TuBB-9-ICG 2, and NLS-TuBB-9-ICG 2 excited by $730 \mathrm{~nm}$ irradiation. The fluorescence intensity also decreased slightly after the NLS conjugation since the number of ICG molecules per antibody decreased. 

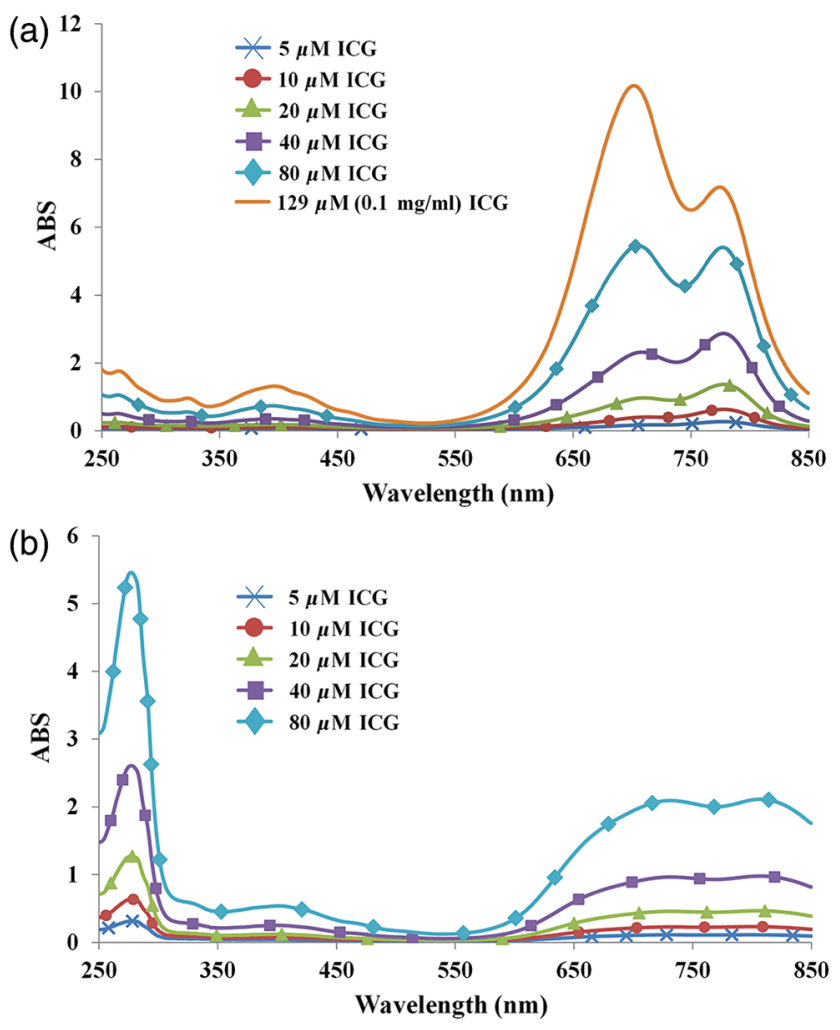

Fig. 2 Absorption spectra of (a) free ICG and (b) ICG linked to the antibody TuBB-9 (ratio $1: 1.8$ ) in $\mathrm{H}_{2} \mathrm{O}$ at different concentrations. Free ICG shows a characteristic absorption band exhibiting two distinct peaks in the NIR. Conjugation to the antibody leads to a broadening of the absorption peaks. The additional peak at $280 \mathrm{~nm}$ originates from protein absorption of the TuBB-9 antibody.

\subsection{Nucleolar Localization of NLS-TUBB-9-ICG after Photochemical Internalization Irradiation}

After incubation of HeLa and OVCAR-5 cells with NLS-TuBB9-ICG, constructs are taken up by the cells and are entrapped in endosomal structures (Fig. 4, middle panel). Entrapped constructs were released to the cytosol by the PCI process. Cells were first incubated with BPD and then irradiated at $410 \mathrm{~nm}$. At $410 \mathrm{~nm}$ the photosensitizer BPD is efficiently excited without activating ICG, which exhibits a maximum absorption at $700 \mathrm{~nm}$. Figure 4 shows the intracellular distribution of NLSTuBB-9-ICG before and $48 \mathrm{~h}$ after PCI irradiation.

Without PCI, both HeLa and OVCAR-5 cells showed a dotted fluorescence pattern in the cytoplasm after staining TuBB-9 with a secondary antibody. This indicates the trapping of NLSTuBB-9-ICG in endosomal structures. In contrast, $48 \mathrm{~h}$ after PCI irradiation fluorescence was detected uniformly spread over the cytoplasm and also located in the nucleoli at more distinct sites, which indicates the release of NLS-TuBB-9-ICG into the cytoplasm and binding to the $\mathrm{Ki}-67$ protein. A similar relocalization from the cytosol to the nucleus was observed after cytosolic microinjection of TuBB-9-FITC conjugates in earlier experiments. ${ }^{26}$ Approximately, $90 \%$ of HeLa cells growing as monolayer cell culture are positive for Ki-67. ${ }^{28}$ This means that at the maximum $90 \%$ of the cells can show nucleolar localization of the TuBB-9 constructs. In earlier studies we have shown that with NLS-TuBB-9-FITC nucleolar localization was achieved in $78 \%$ of Ki-67 positive cells. ${ }^{28}$
At the time point of the second irradiation for the Ki-67 inactivation ( $72 \mathrm{~h}$ after incubation start), the BPD fluorescence is not detectable anymore in HeLa or in OVCAR-5 cells (Fig. 5).

\subsection{In Vitro Ki-67 Photo-Inactivation with Indocyanine Green Conjugates}

In previous studies, we demonstrated the ability of NLS-TuBB9-FITC to kill various proliferating cells after the dual irradiation with wavelengths of 690 and $490 \mathrm{~nm} \cdot{ }^{27,28}$ The irradiation of NLS-TuBB-9-FITC leads to cell death by the inactivation of the $\mathrm{Ki}-67$ protein or a binding partner. In order to evaluate the efficiency of the ICG constructs exerting their action after the dual irradiation with wavelength of 410 and $690 \mathrm{~nm}$, an in vitro cytotoxicity assay was performed. Figure 6(a) shows the cytotoxic effects of different NLS-TuBB-9-ICG constructs in comparison with NLS-TuBB-9-FITC on HeLa cells. Untreated cells and cells incubated only with BPD served as controls. Due to damaging effects to endosomal membranes, all groups treated with BPD showed a loss in cell viability of approximately $20 \%$ to $30 \%$ after 410 or $690 \mathrm{~nm}$ irradiation alone. However, groups treated with NLS-TuBB-9-FITC or NLS-TuBB-9-ICG and irradiated at 410 and $690 \mathrm{~nm}$ showed a considerably higher cell killing compared to the groups of untreated cells and cells incubated with BPD but without irradiation. Differences in cell viability were observed between the different NLS-TuBB-9-ICG constructs and NLS-TuBB-9-FITC. Among the three different ICG constructs, the NLS-TuBB-9ICG (ratio 1:1.8) showed the highest cell killing efficiency (cell viability of $13.7 \% \pm 3.1 \%$ ), compared to the group treated with the NLS-TuBB-9-ICG (ratio $1: 0.6)(29.2 \% \pm 5.8 \%)$ or with NLS-TuBB-9-ICG (ratio $1: 5.2)(22.4 \% \pm 2.6 \%)$. No significant difference was observed between NLS-TuBB-9-ICG (ratio $1: 1.8$ ) and NLS-TuBB-9-FITC.

The treatment procedure was also applied on ovarian carcinoma cells OVCAR-5 using NLS-TuBB-9-ICG (ratio $1: 1.8$ ) and NLS-TuBB-9-FITC conjugates. Cytotoxic effects were comparable, but slightly lower, compared with HeLa cells [Fig. 6(b)].

\section{Discussion}

In our study, we demonstrated the ability of cell penetrating ICG-antibody-conjugates to inactivate the Ki-67 protein. ICG is routinely used in angiography or as a contrast agent in cancer surgery and has an excellent safety profile. The broad absorption in the NIR allows for a large range of PDT applications of ICG. Although phototoxic effects were described, the use of unbound ICG is not very common for PDT. This is partly due to the aggregation pattern that leads to a subsequent quenching of the excited state with a reduction of singlet oxygen production.

We linked ICG covalently in different ratios to the Ki-67 binding antibody TuBB-9, which is the only one that recognizes $\mathrm{Ki}-67$ in an active state. All ICG antibody conjugates showed a linear increase of absorption with concentration in a relevant range. Obviously, the binding to macromolecular antibodies prevents aggregation of the dye. Furthermore, fluorescence is not compromised by the conjugation to NLS sequences. A slight decrease of ICG fluorescence is caused by a replacement of ICG molecules by the NLS sequence on the antibody.

With the help of the NLS peptides and the recently developed two step light controlled delivery of antibody conjugates into 
(a)

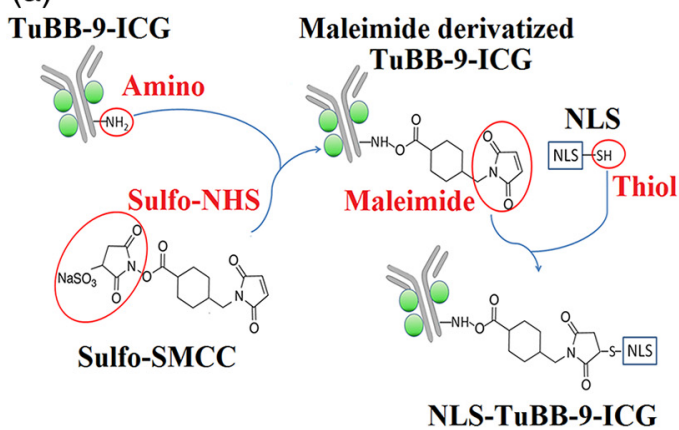

(c)

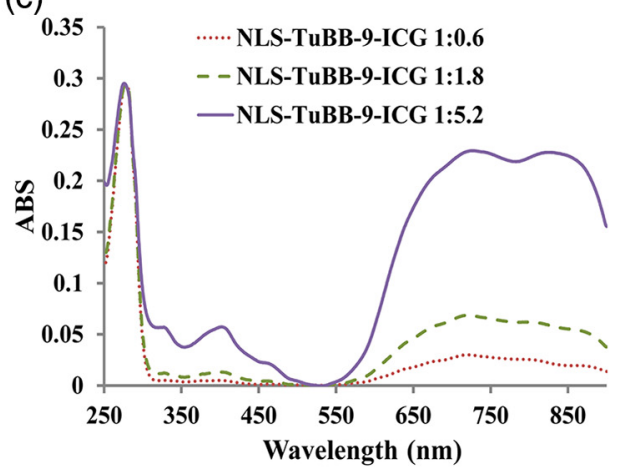

(b)

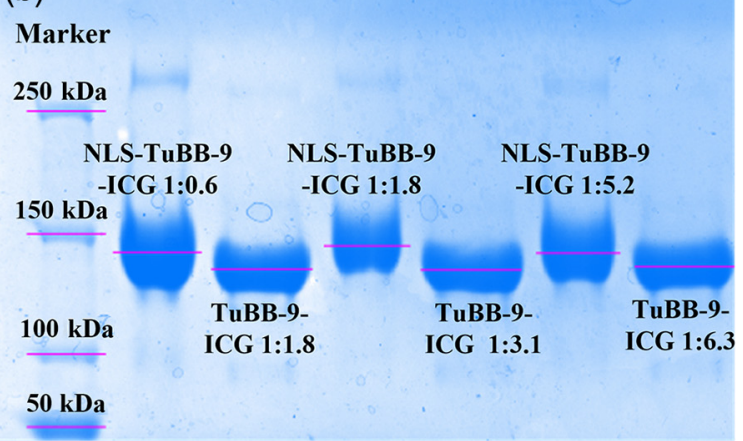

(d) Emission spectrum under 730 nmexcitation

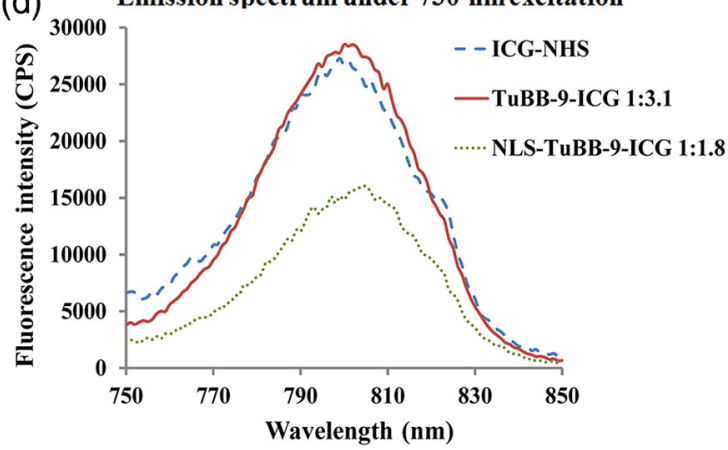

Fig. 3 (a) Conjugation scheme of TuBB-9-ICG with NLS. TuBB-9-ICG conjugate was incubated with an SMCC-linker that binds with its NHS group to the amino group of the antibody. In the next step, the maleimide group of the linker reacts with thiols on the NLS peptide to form TuBB-9-ICG-NLS. (b) Characterization of the antibody conjugates with SDS-PAGE and coomassie staining. (c) Absorption spectrum of the NLS-TuBB-9-ICG constructs and (d) fluorescence spectrum of ICG-NHS, TuBB-9-ICG 2, and NLS-TuBB-9-ICG 2. All samples are normalized to the TuBB-9 antibody concentration.

Table 2 Characterization of NLS-TuBB-9-ICG constructs.

\begin{tabular}{|c|c|c|c|}
\hline $\begin{array}{l}\text { Name of NLS } \\
\text { constructs }\end{array}$ & $\begin{array}{c}\text { Molar ratio of } \\
\text { TuBB-9 and } \\
\text { ICG before NLS } \\
\text { conjugation }\end{array}$ & $\begin{array}{l}\text { Molar ratio of } \\
\text { TuBB-9 and } \\
\text { ICG after NLS } \\
\text { conjugation }\end{array}$ & $\begin{array}{c}\text { Estimated } \\
\text { amount of } \\
\text { NLS-peptides } \\
\text { conjugated to } \\
\text { TuBB-9-ICG } \\
\end{array}$ \\
\hline NLS-TuBB-9-ICG 1 & $1: 1.8$ & $1: 0.6$ & 5 \\
\hline NLS-TuBB-9-ICG 2 & $1: 3.1$ & $1: 1.8$ & 6 \\
\hline NLS-TuBB-9-ICG 3 & $1: 6.3$ & $1: 5.2$ & 3 \\
\hline
\end{tabular}

the cytoplasm, we were able to specifically deliver TuBB-9ICG-NLS constructs to the cell nucleoli, where the Ki-67 protein is located. After intracellular uptake, macromolecules are often entrapped in endosomal structures of the cells. We successfully used PCI with the photosensitizer BPD to disrupt the endosomes. For many PCI applications, amphiphilic dyes are used, which bind to cellular membranes. BPD is a lipophilic Porphyrine and for HeLa and OVCAR-5 cells it worked as a good PCI sensitizer. ${ }^{28}$ Since the aim of this study is the investigation of the potential of ICG for protein inactivation, we irradiated BPD at $410 \mathrm{~nm}$ where the absorption of ICG is low, to prevent activation and bleaching of ICG. After irradiation of BPD we observed localization of TuBB-9-ICG-NLS in the nucleoli. We have shown in earlier studies, that with NLS-
TuBB-9-FITC nucleolar localization was achieved in $78 \%$ of Ki-67 positive cells. ${ }^{28}$ Nucleolar localization of the antibody is presumably due to cotransport of the antibody with newly synthesized Ki-67 protein from the cytoplasm to the nucleus, or due to binding to Ki-67 during mitosis after breakdown of the nuclear envelope. After a second light irradiation at $690 \mathrm{~nm} 48 \mathrm{~h}$ later cells showed a pronounced cell death. The time delay ensured effective nucleolar localization of the ICG conjugates in most of the cells. The cell death is selectively provoked by irradiation of ICG bound to the TuBB-9 antibody, since BPD was not detectable anymore in the cell at the time point of the second irradiation. Samples incubated only with BPD showed no significant increase in cell death after the second irradiation with $690 \mathrm{~nm}$. Although the overall strategy is complex, BPD and ICG and their light-irradiation are clinically approved.

Light inactivation of Ki-67 with the antibody TuBB-9 leads to cell death. The inactivation of Ki-67 is most likely due to photochemical crosslinking with close-by interacting proteins. This mechanism is also proposed for the established method called chromophore assisted light inactivation where the oxidation of methionine side chains has been shown. ${ }^{39-41}$

An antibody ICG ratio of $1: 1.8$ showed the highest amount of cell death compared to the other ratios of $1: 0.6$ and $1: 5.2$. The conjugation ratio of 1:0.6 was less effective most likely due to the lower number of dye molecules that are available for inactivation. When the number of dye molecules per antibody molecule increases over the ratio of $1: 1.8$, it might hinder the antibody to bind to its target protein or self-quenching may 


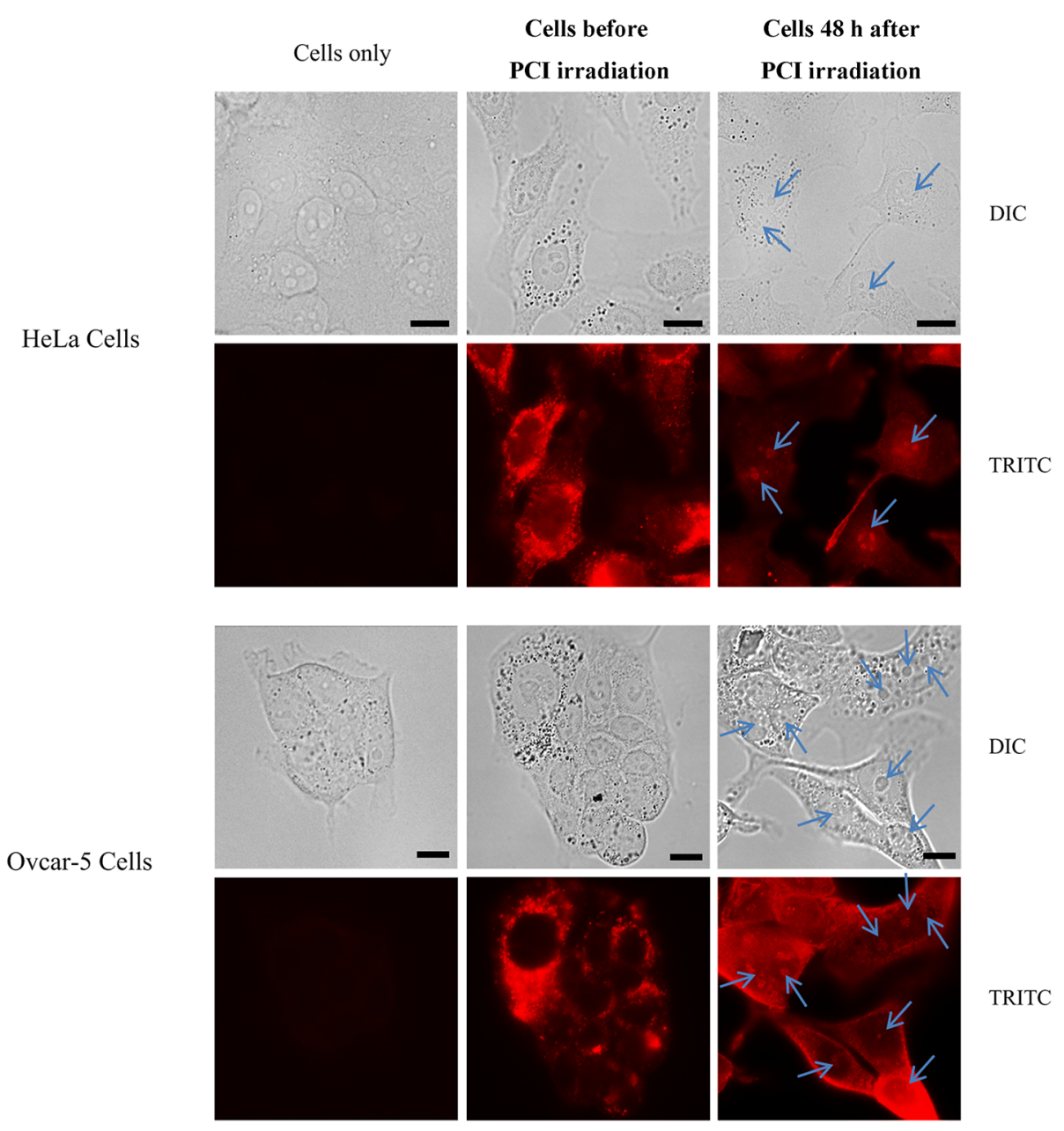

Fig. 4 Nucleolar localization of NLS-TuBB-9-ICG was observed in HeLa and OVCAR- 5 cells $48 \mathrm{~h}$ after BPD mediated photochemical internalization. Cells were incubated with BPD for $18 \mathrm{~h}$ and then for $4 \mathrm{~h}$ with NLS-TuBB-9-ICG. Before PCI irradiation, TuBB-9 conjugates located in endosomal structures in the cytoplasm. $48 \mathrm{~h}$ after irradiation at $410 \mathrm{~nm}$ for activation of BPD conjugates locate also in the nucleoli of the cells at the site of Ki-67. TuBB-9 was stained by the secondary antibody goat-anti-mouse Alexa 546. Arrows in fluorescence images point to the localization of the nucleoli. Size bars: $10 \mu \mathrm{m}$.
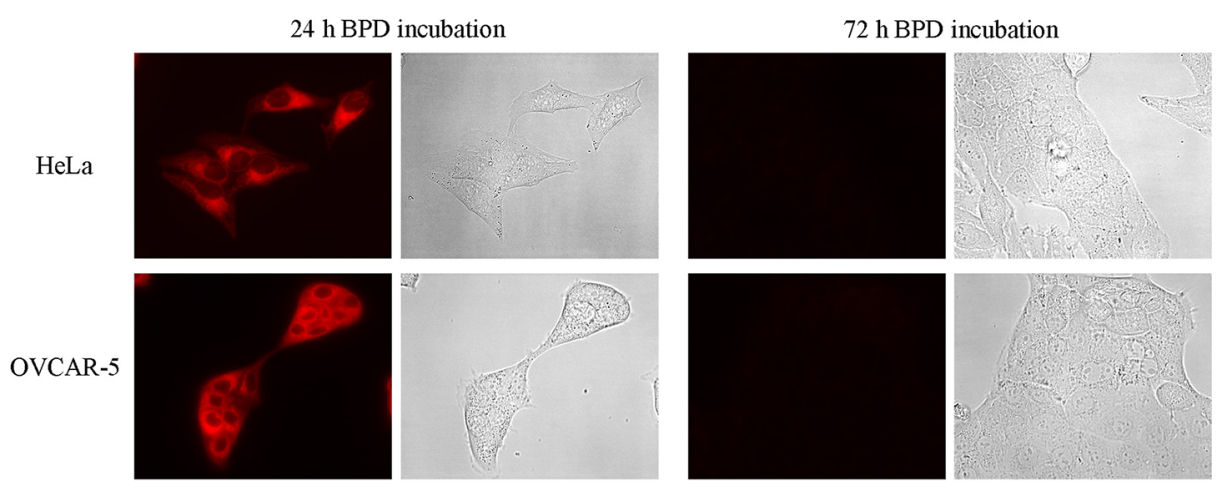

Fig. 5 HeLa cells and OVCAR- 5 cells incubated with $100 \mathrm{nM}$ BPD. After $24 \mathrm{~h}$ incubation, bright BPD fluorescence can be detected under the fluorescence microscope. $72 \mathrm{~h}$ after incubation start the BPD fluorescence is not detectable. Illumination, exposure times and objective are identical in all images.

occur due to the close proximity of the dye molecules. Interestingly, these 1:1.8 TuBB-9-ICG-NLS constructs led to a similar extent of cell death compared with TuBB-9-FITC-NLS. From this, we conclude that ICG as antibody conjugate has a strong potential in molecular targeted PDT applications, where light absorption in the NIR is inevitable for good light penetration into the tissue. The advantage of our approach of Ki-67 inactivation is the effectiveness of cell elimination with 

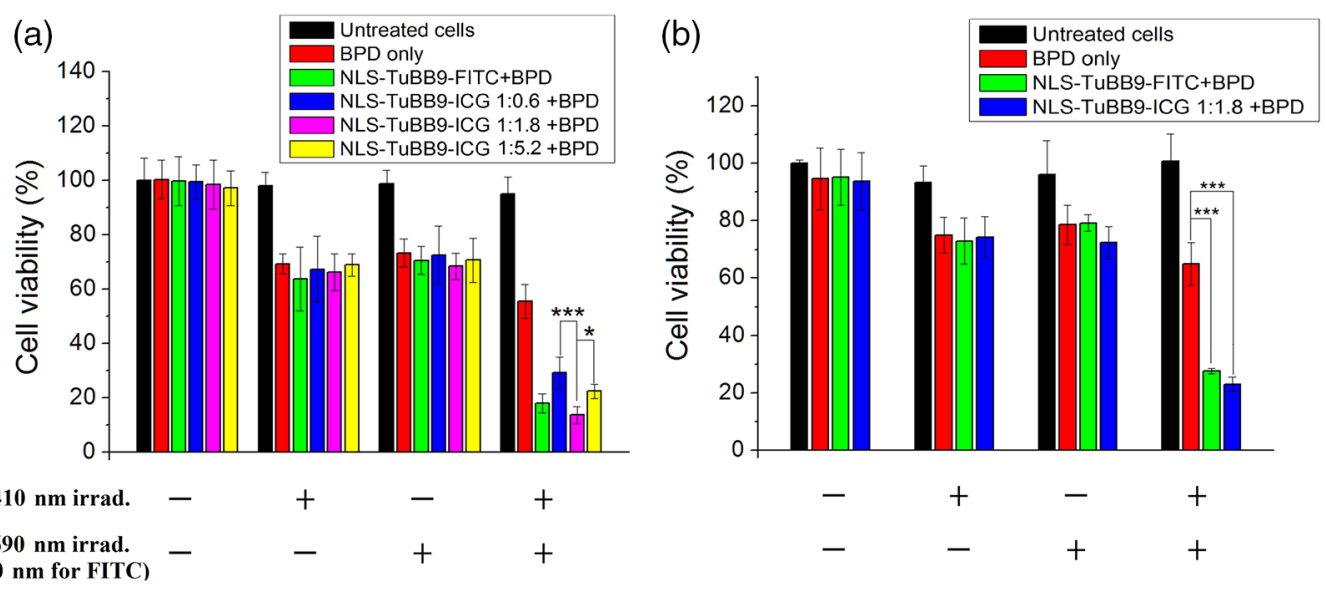

Fig. 6 NLS-TUBB-9-ICG constructs eliminated effectively proliferating (a) HeLa and (b) OVCAR-5 cells after 410 and $690 \mathrm{~nm}$ irradiation. Irradiation at $410 \mathrm{~nm}$ caused endosomal release and irradiation at $690 \mathrm{~nm}$ lead to the inactivation of $\mathrm{Ki}-67$. The middle columns show cells that got only irradiation with one wavelength and demonstrate unspecific cell killing. All NLS-TuBB-9-ICG constructs showed effective cell elimination, but best results were obtained with NLS-TUBB-9-ICG 2. ${ }^{* * *}$ indicates statistically significant difference in cell viability between two linked samples after t-test analysis $(p<0.05)$. (B) MTT assay reveals the capacity of the NLS-TuBB-9-ICG 2 for elimination of proliferating OVCAR- 5 cells.

low antibody and dye concentration in the nanomolar range. This effectiveness is combined with high selectivity for proliferating cells. The light irradiation adds an additional level of selectivity, since the antibody itself does not inhibit Ki-67. This approach can also be applied on other membrane or cellular target proteins for light triggered inactivation. The use of ICG allows the inactivation deeper in the tissue, which is important for further in vivo applications.

\section{Conclusion}

Our results show that ICG antibody conjugates are effective photosensitizers for molecular targeted PDT. Since it is FDA approved for many applications and can be useful for biomedical applications due to its absorption characteristics in the NIR, a rapid clinical use seems feasible.

\section{Acknowledgments}

This work was supported by the German Research Foundation (DFG, grant no. Ra1771/3-1) and the National Natural Science Foundation of China (grant no. 61120106013).

\section{References}

1. J. T. Alander et al., "A review of indocyanine green fluorescent imaging in surgery," J. Biomed. Imaging 2012, 940585 (2012).

2. R. Weissleder, "A clearer vision for in vivo imaging," Nat. Biotechnol. 19(4), 316-317 (2001).

3. R. Sharma et al., "New horizons for imaging lymphatic function," Ann. N.Y. Acad. Sci. 1131(1), 13-36 (2008).

4. B. E. Schaafsma et al., "The clinical use of indocyanine green as a nearinfrared fluorescent contrast agent for image-guided oncologic surgery," J. Surg. Oncol. 104(3), 323-332 (2011).

5. J. A. Zelken and A. P. Tufaro, "Current trends and emerging future of indocyanine green usage in surgery and oncology: an update," Ann. Surg. Oncol. 22 Suppl 3, 1271-1283 (2015).

6. N. Kokudo and T. Ishizawa, "Clinical application of fluorescence imaging of liver cancer using indocyanine green," Liver Cancer 1(1), 15-21 (2012).

7. A. Bille et al., "Preoperative intrathoracic lymph node staging in patients with non-small-cell lung cancer: accuracy of integrated positron emission tomography and computed tomography," Eur. J. CardioThoracic Surg. 36(3), 440-445 (2009).

8. T. Hasan et al., "Photodynamic therapy of cancer," Cancer Med. 7, 537548 (2003).

9. M. Price et al., "Monitoring singlet oxygen and hydroxyl radical formation with fluorescent probes during photodynamic therapy," Photochem. Photobiol. 85(5), 1177-1181 (2009).

10. C. Abels et al., "Indocyanine green (ICG) and laser irradiation induce photooxidation," Arch. Dermatol. Res. 292(8), 404-411 (2000).

11. W. Bäumler et al., "Photo-oxidative killing of human colonic cancer cells using indocyanine green and infrared light," $B r$. J. Cancer 80(3-4), 360-363 (1999).

12. E. Crescenzi et al., "Photodynamic therapy with indocyanine green complements and enhances low-dose cisplatin cytotoxicity in MCF-7 breast cancer cells," Mol. Cancer Ther. 3(5), 537-544 (2004).

13. O. Bozkulak et al., "Photo-toxic effects of 809-nm diode laser and indocyanine green on MDA-MB231 breast cancer cells," Photodiagn. Photodyn. Ther. 6(2), 117-121 (2009).

14. W. W. Tseng et al., "Infrared laser activation of indocyanine green inhibits growth in human pancreatic cancer," Pancreas 27(3), e42-e45 (2003).

15. H.-J. Lim and C.-H. Oh, "Indocyanine green-based photodynamic therapy with $785 \mathrm{~nm}$ light emitting diode for oral squamous cancer cells," Photodiagn. Photodyn. Ther. 8(4), 337-342 (2011).

16. A.-M. Mamoon et al., "In vitro efficiency and mechanistic role of indocyanine green as photodynamic therapy agent for human melanoma," Photodiagn. Photodyn. Ther. 6(2), 105-116 (2009).

17. E. M. Sevick-Muraca, "Translation of near-infrared fluorescence imaging technologies: emerging clinical applications," Аппи. Rev. Med. 63, 217-231 (2012).

18. F. Yan et al., "Molecular imaging-guided photothermal/photodynamic therapy against tumor by iRGD-modified indocyanine green nanoparticles," J. Controlled Release 224, 217-228 (2016).

19. L. Liu et al., "An activatable theranostic nanomedicine platform based on self-quenchable indocyanine green-encapsulated polymeric micelles," J. Biomed. Nanotechnol. 12(6), 1223-1233 (2016).

20. W. S. Kuo et al., "Gold nanomaterials conjugated with indocyanine green for dual-modality photodynamic and photothermal therapy," Biomaterials 33(11), 3270-3278 (2012).

21. A. Sarkar et al., "Photocatalytic activity of $\mathrm{TiO}_{2}$ nanoparticles: effect of thermal annealing under various gaseous atmospheres," Nanotechnology 23(47), 475711 (2012).

22. J. Gerdes et al., "Cell cycle analysis of a cell proliferation-associated human nuclear antigen defined by the monoclonal antibody Ki-67," J. Immunol. 133(4), 1710-1715 (1984). 
23. K. Sasaki et al., "The cell cycle associated change of the Ki-67 reactive nuclear antigen expression," J. Cell. Physiol. 133(3), 579-584 (1987).

24. T. Scholzen and J. Gerdes, "The Ki-67 protein: from the known and the unknown," J. Cell. Physiol. 182(3), 311-322 (2000).

25. J. Bullwinkel et al., "Ki-67 protein is associated with ribosomal RNA transcription in quiescent and proliferating cells," J. Cell. Physiol. 206(3), 624-635 (2006).

26. R. Rahmanzadeh et al., "Chromophore-assisted light inactivation of pKi-67 leads to inhibition of ribosomal RNA synthesis," Cell Proliferation 40(3), 422-430 (2007).

27. R. Rahmanzadeh et al., "Ki-67 as a molecular target for therapy in an in vitro three-dimensional model for ovarian cancer," Cancer Res. 70(22), 9234-9242 (2010).

28. S. Wang et al., "Light-controlled delivery of monoclonal antibodies for targeted photoinactivation of Ki-67," Mol. Pharmaceutics 12(9), 32723281 (2015).

29. S. Wang et al., "A light-controlled switch after dual targeting of proliferating tumor cells via the membrane receptor EGFR and the nuclear protein Ki-67," Sci. Rep. 6, 27032 (2016).

30. M. Landsman et al., "Light-absorbing properties, stability, and spectral stabilization of indocyanine green," J. Appl. Physiol. 40(4), 575-583 (1976).

31. Y.-Z. Lin et al., "Inhibition of nuclear translocation of transcription factor NF- $\kappa$ B by a synthetic peptide containing a cell membrane-permeable motif and nuclear localization sequence," J. Biol. Chem. 270(24), 14255-14258 (1995).

32. H. K. Shete, R. H. Prabhu, and V. B. Patravale, "Endosomal escape: a bottleneck in intracellular delivery," J. Nanosci. Nanotechnol. 14(1), 460-474 (2014).

33. R. Ezzeddine et al., "Effect of molecular characteristics on cellular uptake, subcellular localization, and phototoxicity of $\mathrm{Zn}$ (II) N-alkylpyridylporphyrins," J. Biol. Chem. 288(51), 36579-36588 (2013).

34. P. K. Selbo et al., "Photochemical internalization provides time-and space-controlled endolysosomal escape of therapeutic molecules," J. Controlled Release 148(1), 2-12 (2010).

35. B. Aveline, T. Hasan, and R. W. Redmond, "Photophysical and photosensitizing properties of benzoporphyrin derivative monoacid ring A (BPD-MA)," Photochem. Photobiol. 59(3), 328-335 (1994).

36. J. Moan and K. Berg, "The photodegradation of porphyrins in cells can be used to estimate the lifetime of singlet oxygen," Photochem. Photobiol. 53(4), 549-553 (1991).

37. L. Prasmickaite et al., "Photochemical disruption of endocytic vesicles before delivery of drugs: a new strategy for cancer therapy," $B r . J$. Cancer 86(4), 652-657 (2002).

38. P. Chen et al., "Nuclear localizing sequences promote nuclear translocation and enhance the radiotoxicity of the anti-CD33 monoclonal antibody HuM195 labeled with 111In in human myeloid leukemia cells," J. Nucl. Med. 47(5), 827-836 (2006).

39. P. Yan et al., "Fluorophore-assisted light inactivation of calmodulin involves singlet-oxygen mediated cross-linking and methionine oxidation," Biochemistry 45, 4736-4748 (2006).

40. J. C. Liao, J. Roider, and D. G. Jay, "Chromophore-assisted laser inactivation of proteins is mediated by the photogeneration of free radicals," Proc. Natl. Acad. Sci. 91(7), 2659-2663 (1994).
41. Y. Sano, W. Watanabe, and S. Matsunaga, "Chromophore-assisted laser inactivation-towards a spatiotemporal-functional analysis of proteins, and the ablation of chromatin, organelle and cell function," J. Cell Sci. 127(Pt 8), 1621-1629 (2014).

Sijia Wang received his $\mathrm{PhD}$ in biomedical engineering from Xi'an Jiaotong University in 2016. From 2013 to 2015, he worked as a joint PhD student at the Institute of Biomedical Optics, University of Lübeck in Germany supported by the China Scholarship Council. $\mathrm{He}$ is working on research of biomedical optics including biomedical application of gold nanoparticles and photodynamic therapy.

Gereon Hüttmann received his MSc degree in physics and his $\mathrm{PhD}$ in physical chemistry. From 1992 to 2005, he was a research member and scientific group leader of the Medical Laser Center in Lübeck, Germany. Since 2005, he has been leading a research group at the Institute of Biomedical Optics, University of Lübeck. His main research interests are photothermal and photochemical microeffects, optical coherence tomography, and multiphoton microscopy.

Florian Rudnitzki received his MS degree in biomedical engineering from the University of Lübeck, Germany, in 2008. He focused his studies on pulsed laser irradiated gold nanoparticles for the application of cell manipulation and elimination. He continued his work in the group of $\mathrm{Dr}$. Hüttmann as a $\mathrm{PhD}$ candidate on single particle detection and measurement of laser generated vapor bubbles around gold nanoparticles, providing a basic research for application in platforms and tools for cytometry.

Heyke Diddens-Tschoeke received her $\mathrm{PhD}$ in biology from the University Tübingen, Germany, in 1979. She conducted research on mechanisms of resistance of human tumor cells against anticancer drugs. Since her appointment as senior scientist at the Medical Laser Center/BMO in Lübeck in 1988, her work has focused on different aspects of experimental photodynamic therapy, including pulsed photosensitization, photoresistance, influence of photodynamic therapy on the metastatic potential of tumor cells, antimicrobial photodynamic therapy, and dye-enhanced photothermal therapy.

Zhenxi Zhang received his $\mathrm{PhD}$ in biomedical engineering from Xi'an Jiaotong University in 1990. He has been a researcher multiple times in Germany, including the University of Stuttgart, GSF-National Research Center for Environment and Health, Humboldt University, Wilhelmshaven University of Applied Sciences, and the University of Lübeck. He is a Professor at the Xi'an Jiaotong University; his group focuses on applied technology in biomedical optics, optical biophysics, biomedical optical imaging processes, and spectral analysis methods.

Ramtin Rahmanzadeh received his diploma in biology from the University of Bonn and his PhD from the Leibniz-Research-Center Borstel, Germany. He conducted his postdoctoral research in Tayyaba Hasans laboratory at the Wellman Center for Photomedicine, Harvard Medical School, before starting a laboratory at the University of Lübeck. The focus of his work is the light-controlled manipulation of proteins and cells for the development of molecular targeted approaches for basic research and for photodynamic therapy. 